ANNALES

POLONICI MATHEMATICI

IX (1960)

\title{
Differential inequalities with unbounded operators in Banach spaces
}

\author{
by W. MraK (Kraków)
}

The present paper attempts to give an abstract formulation of theorems concerning differential inequalities. We apply the Hille-Yosida theory of semi-groups of linear bounded operators (see for instance [7]). More precisely, we deal with positivity-preserving semi-groups. Such semi-groups have been considered in connection with temporally homogeneous stochastic processes of Markoff type. For details in this field we refer to [3], [4], [5], [6], [17]. It must be remarked that infinitesimal generators of positivity-preserving semi-groups in concrete functional spaces of continuous or summable functions are in a certain sense second order elliptic operators possessing the maximum property (see [4], [15] and [18]). Thus the theory developed in the present paper combined with the results of the papers mentioned above gives immediately an operator-theoretical treatment of parabolic differential inequalities. Our theorems make it possible to consider countable systems of differential inequalities in the space $(l)$. It is sufficient in this case to combine our theorems with the theory of integration of Kolmogoroff equations of Markoff processes with a countable number of possible states (see [8]). However, a more detailed study in that field may be conducted by the application of methods similar to that used in [6] and [12].

In our investigations we make use of some theorems similar to the generalized mean value theorem (see [2], [11] and [16]). The relation of inequality is defined by means of a positive cone (see [7]).

For the terminology and some simple properties of semi-groups of operators we refer to [7].

We discuss linear differential inequalities in sections 2,3 and 4 . Section 5 concerns some simple almost linear differential inequalities.

1. Let $E$ be a Banach space. The norm of $x \in E$ is denoted by $|x|$. By $\xi, \eta, \ldots$ we denote continuous and linear functionals over $E$. The totality of such functionals, i. e. the adjoint space of $E$ is denoted by $E^{*}$. The real-valued functions of the real variable $t$ are denoted by small Greek letters $\varphi, \psi, \sigma, \ldots$ etc. The operator $A$ is linear if it is additive and 
homogeneous. The domain of $A$ is denoted by $D[A]$. We deal only with operators whose domain and range are included in the same space $E$.

$S$ is a cone if the following conditions hold:

$(\alpha)$ if $x \in S$ and $y \in S$ then $x+y \in S$

( $\beta$ if $\lambda \geqslant 0$ and $x \in S$ then $\lambda x \in S$;

$(\gamma) S$ is closed.

We define the relation " $\leqslant$ " as follows:

$$
x \leqslant y \stackrel{\text { def }}{\equiv} y-x \in S .
$$

Let $S^{*}$ be the set of all functionals $\xi \in E^{*}$ such that $\xi x \geqslant 0$ for each $x \in S$. The lemma below follows immediately from the well known results of Mazur [10]:

LEMma 1. Suppose that for every $\xi \in S^{*}$ the inequality $\xi x \geqslant 0$ holds. Then $x \in S$.

It is thus seen that $S$ may be identified with the set of $x$-es satisfying $\xi x \geqslant 0$ with suitable $\xi$

A linear operator is called positive if $B x \geqslant \theta$ for $x \in S \cap D[B]$. We say that a certain condition holds nearly everywhere [19] if it holds overywhere except an at most denumerable set of points. For the sake of clarity we use in the following only the right-hand upper Dini derivative $\bar{D}_{+}$. However, the properties discussed remain true if $\bar{D}_{+}$is replaced by any other Dini derivative.

We now establish some notational conventions. The symbol w-lim denotes a weak limit, $\mathrm{s}$-lim a strong one. Let $x(t)$ be defined in a neighborhood of $t_{0}$. We define

$$
\begin{aligned}
D_{+}^{w} x\left(t_{0}\right) & =\mathrm{w}-\lim _{h \rightarrow 0+} \frac{x\left(t_{0}+h\right)-x\left(t_{0}\right)}{h}, \\
D_{+}^{s} x\left(t_{0}\right) & =\mathrm{s}-\lim _{h \rightarrow 0+} \frac{x\left(t_{0}+h\right)-x\left(t_{0}\right)}{h}, \\
x^{\prime}\left(t_{0}\right) & =\mathrm{s}-\lim _{h \rightarrow 0} \frac{x\left(t_{0}+h\right)-x\left(t_{0}\right)}{h} .
\end{aligned}
$$

The right-hand weak (strong) partial differentiation is denoted by $\partial_{+}^{w} / \partial s$ $\left(\partial_{+}^{s} / \partial s\right)$. The bilateral strong derivative is denoted by $\partial^{s} / \partial s$. The Bochner integral of $x(t)$ is shortly written as $\int_{a}^{b} x(\tau) d \tau$; the Pettis integral is denoted by (P) $\int_{a}^{b} x(\tau) d \tau$.

It follows from Zygmund's lemma that if $\varphi(t), \psi(t)$ are continuous and satisfy nearly everywhere the inequality $\bar{D}_{+} \varphi(t) \leqslant \psi(t)$, then $\varphi\left(\tau_{1}\right)-\varphi\left(\tau_{2}\right) \leqslant \int_{\tau_{2}}^{\tau_{1}} \psi(\tau) d \tau$. For $\varphi(t)$ absolutely continuous and $\psi(t)$ summable, the inequality ${ }^{\prime} \varphi^{\prime}(t) \leqslant \psi(t)$ satisfied almost everywhere implies that $\varphi\left(\tau_{1}\right)-\varphi\left(\tau_{2}\right) \leqslant \int_{\tau_{1}}^{\tau_{2}} \psi(\tau) d \tau$. It is thus seen that the lemma below follows from lemma 1 combined with the definition of the Pettis integral (see for instance [7]).

LEMMa 2. Suppose that $x(t)$ and $y(t)$ satisfy one of the following conditions:

(1) The function $x(t)$ is weally continuous and for every $\xi \in \mathcal{S}^{*}$ there exists an at most denumerable set $Z_{\xi} \subset(0, \alpha)$ such that $\bar{D}_{+} \xi x(t) \leqslant \xi y(t)$ for $t \epsilon(0, \alpha)-Z_{\xi}$. The function $y(t)$ is weakly continuous and Pettis integrable.

(2) For every $\xi \in S^{*}$ the function $\xi x(t)$ is absolutely continuous and $\frac{d}{d t} \xi x(t)$ $\leqslant \xi y(t)$ for $t \epsilon(0, \alpha)-Z_{\xi}, \operatorname{mes} Z_{\xi}=0$. The function $y(t)$ is Pettis integrable.

Then

$$
x\left(t_{2}\right)-x\left(t_{1}\right) \leqslant(\mathrm{P}) \int_{t_{1}}^{t_{2}} y(s) d s \quad\left(0<t_{1}<t_{2}\right) .
$$

2. Suppose that we are given a one-parameter family $A(t)(0<t<\alpha)$. of linear operators. Let the family $\{U(t, s)\}$ of linear bounded operators possess the following properties:

(4) $U(t, s)$ is positive for $0<s \leqslant t$.

(5) The strong derivative $\frac{\partial_{+}^{s} U(t, s) x}{\partial s}(s<t)$ exists for $x \in D[A]$ and

$$
\frac{\partial_{+}^{s} U(t, s) x}{\partial s}=-U(t, s) A(s) x .
$$

(6) For every $x \in E$ the function $u(s)=U(t, s) x$ is strongly continuous in $s$ and $U(t, t)=I$.

THEOREM 1. Let $U(t, s)$ satisfy $(4),(5)$ and (6). Suppose that $x(t), y(t)$ are strongly continuous in $(0, \alpha)$. We assume that $x(t) \in D[A(t)](0<t<\alpha)$. Let the inequality $D_{+}^{s} x(t) \leqslant A(t) x(t)+y(t)$ be satisfied nearly everywhere in $(0, \alpha)$. Then

$$
\text { (7) } \quad x(t) \leqslant U(t, s) x(s)+\int_{s}^{t} U(t, \tau) y(\tau) d \tau \quad \text { for } \quad 0<s<t \text {. }
$$

Proof. It follows from (4) that for a fixed $t$ the inequality

$$
U(t, s) D_{+}^{s} x(s) \leqslant U(t, s) A(s) x(s)+U(t, s) y(s)
$$


holds nearly everywhere in $(0, t)$. Using $(6)$ we conclude that $U(t, s) x(s)$ is continuous in $s$. Suppose that $D_{+}^{s} x\left(s_{0}\right)$ exists. It follows from the formula

$$
\begin{aligned}
& \frac{1}{h}\left[U\left(t, s_{0}+h\right) x\left(s_{0}+h\right)-U\left(t, s_{0}\right) x\left(s_{0}\right)\right] \\
& =\frac{1}{h}\left[U\left(t, s_{0}+h\right) x\left(s_{0}\right)-U\left(t, s_{0}\right) x\left(s_{0}\right)\right]+U\left(t, s_{0}+h\right) D_{+}^{s} x\left(s_{0}\right)+ \\
& \quad+U\left(t, s_{0}+h\right) \frac{o(h)}{h}
\end{aligned}
$$

and from (6) that

(9) $\quad\left(\frac{\partial_{+}^{s} U(t, s) x(s)}{\partial s}\right)_{s=s_{0}}=\left(\frac{\partial_{+}^{s} U(t, s) x\left(s_{0}\right)}{\partial s}\right)_{s=s_{0}}+U\left(t, s_{0}\right) D_{+}^{s} x\left(s_{0}\right)$.

By (5), (8) and (9) we get

$$
\frac{\partial_{+}^{s}(U(t, s) x(s))}{\partial s} \leqslant U(t, s) y(s)
$$

nearly everywhere in $(0, t)$. Using (10) and lemma 2 we get

$$
U\left(t, s_{2}\right) x\left(s_{2}\right) \leqslant U\left(t, s_{1}\right) x\left(s_{1}\right)+\int_{s_{1}}^{s_{2}} U(t, \tau) y(\tau) d \tau
$$

for $s_{1}<s_{2}<t$. Let $s=s_{1}$ and $s_{2} \rightarrow t$. (7) now follows from (11).

Some obvious consequences of theorem 1 must now be mentioned.

THEOREM 2. Let $U(t, s)$ satisfy (4), (5) and (6). Let $x_{i}(t), y_{i}(t)(i$ $=1,2)$ be strongly continuous in $(0, \alpha)$. Let the inequalities

$$
\begin{aligned}
& D_{+}^{s} x_{1}(t) \leqslant A(t) x_{1}(t)+y_{1}(t), \\
& D_{+}^{s} x_{2}(t) \geqslant A(t) x_{2}(t)+y_{2}(t)
\end{aligned}
$$

be satisfied nearly everywhere in $(0, \alpha)$. Then

$x_{1}(t)-x_{2}(t) \leqslant U(t, s)\left[x_{1}(s)-x_{2}(s)\right]+\int_{s}^{t} U(t, \tau)\left[y_{1}(\tau)-y_{2}(\tau)\right] d \tau \quad(s \leqslant t)$.

Moreover, if $x_{1}(0) \leqslant x_{2}(0)$ and $y_{1}(t) \leqslant y_{2}(t)$ then $x_{1}(t) \leqslant x_{2}(t)$.

Assumptions (4), (5) and (6) hold for $U(t, s)=T(t-s)$ where $\{T(\tau)\}$ is a positivity-preserving semi-group of class $\left(O_{0}\right): A(t)=A$ and $A$ is the infinitesimal generator of $\{T(\tau)\}$. Other conditions which ensure the existence of $U(t, s)$ satisfying (5) and (6) for time-dependent $A(t)$ are given in [9], [14]. In order to ensure (4) one must assume that the semi-groups generated by $A(t)$ are positive.
3. Suppose now that $A$ is the infinitesimal generator of positivitypreserving semi-group $\{T(u)\}$ of class $(0, A)$. We shall prove the following theorem:

THEOREM 3. Let $x(t)$ be weakly continuous in $(0, \alpha)$, and let the inequality

$$
D_{+}^{w o} x(t) \leqslant A x(t)
$$

be satisfied nearly everywhere in $(0, \alpha)$. Then $x(t) \leqslant T(t-s) x(s)$ for $s<t$.

Proof. Define $x_{\lambda}(t)$ as follows: $x_{\lambda}(t)=\lambda R(\lambda, A) x(t)$. Suppose that $D_{+}^{w} x\left(t_{0}\right)$ exists and let $\xi \in E^{*}$. We have

$$
\xi \frac{x_{\lambda}\left(t_{0}+h\right)-x_{\lambda}\left(t_{0}\right)}{h}=\xi \lambda R(\lambda, A) \frac{x\left(t_{0}+h\right)-x\left(t_{0}\right)}{h} .
$$

But $x\left(t_{0}-h\right)-x\left(t_{0}\right) / h$ tends weakly to $D_{+}^{w} x\left(t_{0}\right)$ and $\xi \lambda R(\lambda, A) \in E^{*}$. Hence $D_{+}^{w} x_{\lambda}\left(t_{0}\right)=\lambda R(\lambda, A) D_{+}^{w} x\left(t_{0}\right)$. The resolvent $R(\lambda, A)$ is positive. We thus infer by (12) that

$$
D_{+}^{w} x_{\lambda}(t) \leqslant A x_{\lambda}(t)
$$

nearly everywhere and consequently

$$
T(t-s) D_{+}^{w} x_{\lambda}(s)-T(t-s) A x_{\lambda}(s) \leqslant \theta
$$

nearly everywhere in $(0, t)$. Observe now that

(15) $\quad\left[T\left(\tau_{2}\right)-T\left(\tau_{1}\right)\right] \lambda R(\lambda, A) x=\int_{\tau_{1}}^{\tau_{2}} T(\tau) \lambda A R(\lambda, A) x d \tau, \quad x \in E$

$\left(0<\tau_{1}<\tau_{2}\right)$. This implies that

(16) $\left|\left[T\left(\tau_{2}\right)-T\left(\tau_{1}\right)\right] \lambda R(\lambda, A) x\right| \leqslant \sup _{\tau} \lambda|T(\tau)||A R(\lambda, A)||x|\left|\tau_{2}-\tau_{1}\right|$,

where sup is taken over an arbitrary compact interval of $(0, a)$ which includes $\tau_{1}$ and $\tau_{2}$. Thus, the operator-valued function $T(t) \lambda R(\lambda, A)$ is continuous in $t(t>0)$ in the uniform operator topology. We will prove now that the derivative $\frac{\partial_{+}^{w} T(t-s) x_{\lambda}(s)}{\partial s}$ exists nearly everywhere and is equal to the difference appearing in the left-hand member of (14). Let $\xi \in B^{*}$ and let $D_{+}^{w} x(t)$ exist at $t=t_{0}$. We have

$$
\begin{aligned}
& \xi \frac{T\left(t-t_{0}-h\right) x_{\lambda}\left(t_{0}+h\right)-T\left(t-t_{0}\right) x_{\lambda}\left(t_{0}\right)}{h} \\
& =-\xi \frac{T\left(t-t_{0}-h\right)-T\left(t-t_{0}\right)}{-h} x_{\lambda}\left(t_{0}\right)+\xi T\left(t-t_{0}-h\right) \frac{x_{\lambda}\left(t_{0}+h\right)-x_{\lambda}\left(t_{0}\right)}{h} .
\end{aligned}
$$


The first member in the right-hand sum tends to $-\xi A T\left(t-t_{0}\right) x_{\lambda}\left(t_{0}\right)$. The second member is equal to

$$
\text { (17) } \begin{aligned}
\xi T\left(t-t_{0}-h\right) \lambda R(\lambda, A) \frac{x\left(t_{0}+h\right)-x\left(t_{0}\right)}{h} \\
=\xi\left[T\left(t-t_{0}-h\right)-T\left(t-t_{0}\right)\right] \lambda R(\lambda, A) \frac{x\left(t_{0}+h\right)-x\left(t_{0}\right)}{h}+ \\
\quad+\xi T\left(t-t_{0}\right) \frac{x_{\lambda}\left(t_{0}+h\right)-x_{\lambda}\left(t_{0}\right)}{h}
\end{aligned}
$$

Suppose that $h_{n} \rightarrow 0+$. The second member of the right-hand sum of (17) tends to $T\left(t-t_{0}\right) D_{+}^{w} x_{\lambda}\left(t_{0}\right)$. It follows from the weak convergence of $\frac{x\left(t_{0}+h_{n}\right)-x\left(t_{0}\right)}{h_{n}}$ that there is an $M>0$ such that $\left|\frac{x\left(t_{0}+h_{n}\right)-x\left(t_{0}\right)}{h_{n}}\right| \leqslant M$. By (16) we thus infer that

(18) $\left|\xi\left[T\left(t-t_{0}-h_{n}\right)-T\left(t-t_{0}\right)\right] \lambda R(\lambda, A) \frac{x\left(t_{0}+h_{n}\right)-x\left(t_{0}\right)}{h_{n}}\right| \leqslant M N h_{n}$

with suitable $N$. From the previous part of the proof and from (18) it follows that $\frac{\partial_{+}^{w}\left(T(t-s) x_{\lambda}(s)\right)}{\partial s}$ exists and is equal to the loft-hand difference of (14). Hence the inequality

$$
\frac{\partial_{+}^{w}\left(T(t-s) x_{\lambda}(s)\right)}{\partial s} \leqslant \theta
$$

holds nearly everywhere in $(0, t)$. Applying (16) one easily proves that $T(t-s) x_{\lambda}(s)$ is weakly continuous in $s$. We thus infer that $T\left(t-s_{2}\right) x_{\lambda}\left(s_{2}\right)$ $\leqslant T\left(t-s_{1}\right) x_{\lambda}\left(s_{1}\right)$ for $s_{1}<s_{2}$. But $s-\lim x_{\lambda}(s)=x(s)$. It follows from the last inequality that $T\left(t-s_{2}\right) x\left(s_{2}\right) \stackrel{\lambda \rightarrow \infty}{\leqslant}\left(t-s_{1}\right) x\left(s_{1}\right)$. Now introduce $\tau=$ $=t-s_{2}$. This loads to the following inequality: $T(\tau) x\left(s_{2}\right) \leqslant T(\tau) T\left(s_{2}-\right.$ $\left.-s_{1}\right) x\left(s_{1}\right)$. Thus

$$
\begin{aligned}
\lambda R(\lambda, A) x\left(s_{2}\right) & =\lambda \int_{0}^{\infty} e^{-\lambda \tau} T(\tau) x\left(s_{2}\right) d \tau \\
& \leqslant \lambda \int_{0}^{\infty} e^{-\lambda \tau} T(\tau) T\left(s_{2}-s_{1}\right) x\left(s_{1}\right) d \tau \\
& =\lambda R(\lambda, A) T\left(s_{2}-s_{1}\right) x\left(s_{1}\right) .
\end{aligned}
$$

On the other hand, $s-\lim _{\lambda \rightarrow \infty} \lambda R(\lambda, A) x=x$ for $x \in E$. The assertion of our theorem follows from $(19)$.
Remark 1. Suppose that $w-\lim x(t)=x(0) \leqslant \theta$. From (16) and from the assertion of theorem 3 it follows that $x_{\lambda}(t) \leqslant T(t-s) x_{\lambda}(s)$, and w- $\lim T(t-s) x_{\lambda}(s)=T(t) x_{\lambda}(0) \leqslant \theta$. Hence $x(t) \leqslant \theta$.

Remark 2. Suppose that (12) is replaced by the following inequality:

$$
D_{+}^{w} x(t) \leqslant A x(t)+y(t) .
$$

We assume that $y(t)$ is weakly continuous and $T(t-s) y(s)$ is supposed to be Pettis integrable in $s$. Then the assertion of theorem 3 may be replaced by

$$
x(t) \leqslant T(t-s) x(s)+(\mathrm{P}) \int_{s}^{t} T(t-\tau) y(\tau) d \tau \quad(s<t) .
$$

We have the following generalization of a theorem of Reuter [13]:

THEOREM 4. Let $A_{1}$ and $A_{2}$ be infinitesimal generators of positivitypreserving semi-groups. $T_{1}(t), T_{2}(t)$ of class $(0, A)$. Suppose that $D\left[A_{1}\right]$ $=D\left[A_{2}\right]=D$ and $A_{1} \leqslant A_{2}$. Then $T_{1}(t) \leqslant T_{2}(t)$ for $t>0$.

Proof. Define $x_{1}(u)=T_{1}(u) x$ and let $x \in S \cap D$. It follows from the inequality $A_{1} \leqslant A_{2}$ that

$$
x_{1}^{\prime}(u)=A_{1} T_{1}(u) x \leqslant A_{2} T_{1}(u) x=A_{2} x_{1}(u) .
$$

By theorem 3 we get

$$
e^{-\lambda s} T_{1}(\tau) T_{1}(s) x \leqslant e^{-\lambda s} T_{2}(\tau) T_{1}(s) x
$$

for $0<\tau<\infty, s>0$. We thus obtain

$$
T_{1}(\tau) \lambda R\left(\lambda, A_{1}\right) x \leqslant T_{2}(\tau) \lambda R\left(\lambda, A_{1}\right) x .
$$

The inequality $T_{1}(\tau) \leqslant T_{2}(\tau)$ is now obtained from the following relations: $\mathrm{s}-\lim _{\lambda \rightarrow \infty} \lambda R\left(\lambda, A_{1}\right) x=x$ for $x \in E, \overline{S \cap D}=S$.

It should be remarked that for semi-groups of class $(0, A)$ the condition

$$
\theta \leqslant R\left(\lambda, A_{1}\right) \leqslant R\left(\lambda, A_{2}\right) \text {. }
$$

implies that $A_{1} \leqslant A_{2}$. If $R\left(\lambda, A_{1}\right)$ and $R\left(\lambda, A_{2}\right)$ satisfy (21), then the inequality $T_{1}(u) \leqslant T_{2}(u)$ follows from the Hille inversion formula:

$$
T_{i}(u) x=\mathrm{s}-\lim _{n \rightarrow \infty}\left\{\frac{n}{u} R\left(\frac{n}{u}, A_{i}\right)\right\}^{n} x, \quad i=1,2 .
$$

4. The aim of this section is to discuss the case where a differential inequality holds almost everywhere. In what follows we assume that the function $x(t)$ possesses the strong derivative $x^{\prime}(t)$ almost everywhere $\left.{ }^{1}\right)$.

(') The strong differentiability is not more restrictive than the weak one:
see [1], th. 4 .


For the sake of clarity it is supposed that $x(t)$ can be expressed as the indefinited Bochner integral of $x^{\prime}(t)$, i. e. $x\left(\tau_{2}\right)-x\left(\tau_{1}\right)=\int_{\tau_{1}}^{\tau_{2}} x^{\prime}(\tau) d \tau$ for $\tau_{1}, \tau_{2} \epsilon(0, \alpha)$. Observe that $x(t)$ becomes now strongly absolutely continuous.

THEOREM 5. Let $A$ be an infinitesimal generator of a positivity-preserving semi-group $\{T(u)\}$ of class $(0, A)$. Suppose that the inequality

$$
x^{\prime}(t) \leqslant A x(t)
$$

holds almost everywhere in $(0, \alpha)$. Then

$$
x(t) \leqslant T(t-s) x(s) \quad \text { for } \quad 0<s<t .
$$

Proof. Just as in the proof of theorem 3 we introduce the function $x_{\lambda}(t)=\lambda R(\lambda, A) x(t)$. It follows from (16) that the function $T(t-s) x_{\lambda}(s)$ is strongly absolutely continuous in $s$ in an arbitrary compact interval of $(0, \alpha)$. Using arguments similar to that used in the proof of theorem 3 we derive from (22) the inequality

$$
\frac{\partial^{s}\left(T(t-s) x_{\lambda}(s)\right)}{\partial s} \leqslant \theta
$$

This inequality holds almost everywhere and $T(t-s) x_{\lambda}(s)$ is absolutely continuous in $s$. By lemma 2 we thus obtain

$$
T\left(t-s_{2}\right) x_{\lambda}\left(s_{2}\right) \leqslant T\left(t-s_{1}\right) x_{\lambda}\left(s_{1}\right) \quad\left(s_{1}<s_{2}\right) .
$$

In order to prove (23) we can now apply a procedure used in the proof of theorem 3.

We will prove the following theorem:

THEOREM 6. Suppose that $A$ is an infinitesimal generator of a positivity-preserving semi-group $\{T(u)\}$ of class $(0, A)$. Let the function $x(t)$ be weakly absolutely continuous and Bochner integrable in any subinterval of $(0, a)$. Assume that $\int_{\tau_{1}}^{\tau_{2}} x(\tau) d \tau \in D[A]$ for $\tau_{1}, \tau_{2} \epsilon(0, \alpha)$. Let $A x(t)$ be Bochner integrable in any subinterval of $(0, a)$. It is supposed that for $\xi \in \mathcal{S}^{*}$ the inequality

$$
\frac{d}{d t} \xi x(t) \leqslant \xi A x(t)
$$

holds for $t \epsilon(0, \alpha)-Z_{\xi}, \operatorname{mes} Z_{\xi}=0$.

Then $x(t)$ satisfies (23).
Proof. Define $x_{h}(t)=\int_{i}^{t+h} x(\tau) d \tau$. The integration of $(24)$ shows that

$$
\xi x((t+h)-x(t)) \leqslant \xi \int_{i}^{t+h} A x(\tau) d \tau: \quad \xi \epsilon S^{*} .
$$

The operator $A$ is closed. It follows from our assumptions and from th. 3.7.12 of [7] that

$$
\xi \int_{i}^{t+h} A x(\tau) d \tau=\xi A x_{h}(t) .
$$

On the other hand, $\frac{d}{d t} x_{h}(t)=x(t+h)-x(t)$ for almost all $t$. Using (25) and (26) we infer that the inequality

$$
\frac{d}{d t} x_{h}(t) \leqslant A x_{h}(t)
$$

holds almost everywhere. Obviously $x_{h}(t)$ satisfies the regularity assumptions needed in theorem 5. Hence $x_{h}(t) \leqslant T(t-s) x_{h}(s)$ and consequently

$$
\frac{1}{h} \xi x_{h}(t) \leqslant \frac{1}{h} \xi T(t-s) x_{h}(s) .
$$

The function $x(t)$ is weakly continuous. We infer therefore that $\frac{1}{h} \xi x_{h}(t)$ $\rightarrow \xi x(t), \frac{1}{h} \xi T(t-s) x_{h}(s) \rightarrow \xi T(t-s) x(s)$. The assertion of the theorem follows now from (27).

Remark 3. Suppose that $x(t)$ is merely weakly continuous. Let $A x(t)$ be weakly continuous. Then (23) holds if $(24)$ is replaced by the following condition: for each $\xi \epsilon S^{*}$ the inequality $\bar{D}_{+} \xi x(t) \leqslant \xi A x(t)$ holds nearly everywhere in $(0, \alpha)$.

5. The purpose of this section is to discuss almost linear differential inequalities. For the sake of simplicity we restrict ourselves to the case of time-independent $A$. Throughout our investigations we assume that $A$ is an infinitesimal generator of a positivity-preserving semi-group $\{T(u)\}$ of class $\left(C_{0}\right)$. We say that $f(t, x)$ defined in $\langle 0, a\rangle \times E$ increases in $x$ if the condition $x_{1} \leqslant x_{2}$ implies that $f\left(t, x_{1}\right) \leqslant f\left(t, x_{2}\right)$. In what follows we suppose that $f(t, x)$ is bounded, say $|f(t, x)|<M$.

THEOREM 7. Let the function $x(t)$ be strongly continuous and let the inequality

$$
D_{+}^{w} x(t) \leqslant A x(t)+f(t, x(t))
$$


be satisfied nearly everywhere in $(0, a)$. Suppose that the transformation $F$ defined by

$$
z(t) \rightarrow \int_{0}^{t} T(t-\tau) f(\tau, z(\tau)) d \tau
$$

is completely continuous when considered in the space $C_{E}\langle 0, \alpha\rangle$. Let the function $f(t, x)$ increase in $x$. Then there exists a solution $y(t)$ of the equation

$$
y(t)=T(t) x(0)+\int_{0}^{t} T(t-\tau) f(\tau, y(\tau)) d \tau
$$

such that

$$
x(t) \leqslant y(t) \text { for } t \in\langle 0, \alpha\rangle .
$$

Proof. (28) implies that

$$
x(t) \leqslant T(t) x(0)+\int_{0}^{t} T(t-\tau) f(\tau, x(\tau)) d \tau .
$$

Suppose that $M=\sup |f|$ and $N=\sup _{\langle 0, a\rangle}|T(t)|$. Let $V$ be defined as follows: $z(\cdot) \epsilon V \equiv z \epsilon C_{E}\langle 0, a\rangle, x(t) \leqslant z(t)$ in $\langle 0, a\rangle$ and $|z(t)| \leqslant N|x(0)|+M$.

$\nabla$ is closed and bounded in $C_{E}\langle 0, \alpha\rangle$. Using (32) and the monotonicity of $f(t, x)$ one easily verifies that $F(V) \subset V$. By Schauder's fixed point theorem we infer that there is a solution $y(t)$ of $(30)$ and $y(\cdot) \in V$. Therefore $x(t) \leqslant y(t)$, q. e. d.

Using the method of successive approximations one easily proves the following theorem:

THEOREM 8. Let $f(t, x)$ be continuous and let it satisfy the condition

$$
\left|f\left(t, x_{1}\right)-f\left(t, x_{2}\right)\right| \leqslant K\left|x_{1}-x_{2}\right| .
$$

Suppose that $f(t, x)$ increases in $x$. Let $x(t)$ be strongly continuous and let $x(t)$ satisfy (28). Then $x(t) \leqslant y(t)$, where $y(t)$ is the unique solution of (30).

\section{References}

[1] A. Alexiewicz, On differentiation of vector-valued functions, Studia Math. 11 (1949), p. 185 - 196.

[2] - On a theorem of Ważewski, Ann. Soc. Polon. Math. 24 (1951), p. $129-131$

[3] W. Feller, The general diffusion operator and positivity preserving semigroups in one dimension, Ann. of Math. 66 (1954) No. 3, p. 417-436.

[4] - On positivity preserving semi-groups of transformations on $O\left[r_{1}, r_{2}\right]$, Ann. Soc Polon.

[5] E. Hille, The abstract Cauchy problem and Oauchy's problem for parabolic equations, Journ. d'Analyse Math. 3 (1953/54), p. 81-196.
[6] - Perturbation methods in the study of Kolmogoroff's equations, Proc. Int. Congres. Math. 1954, Vol. III, Amsterdam 1956, p. 365-376.

[7] - and R. S. Phillips, Functional analysis and semi-groups, Amer. Soc. Col. Publ. 1957.

[8] T. Kato, On the semi-groups generated by Kolmogoroff's differential equa. tions, Journ. Math. Soc. Japan 6 (1954) No. 1, p. 1-15.

[9] - Integration of equation of evolution in Banach space, Journ. Math. Soc. Japan 5 (1953), p. 208 - 234.

[10] S. Mazur, Über konvexe Mengen in linearen normierten Räumen, Studia Math. 4 (1933), p. $70-84$

[11] W. Mlak, Differential inequalities in linear spaces, Ann. Pol. Math. 5 (1958), p. $95-101$.

[12] G. E. H. Reuter and W. Ledermann, On the differential equations for the transition probabilities of Markov processes with enumerably many states, Proc. Cambridge Phil. Soc. 49 (1953), p. $247-262$.

[13] G. E. H. Reuter, A note on contraction semi-groups, Math. Scand. 3.2 (1955), p. $275-280$.

[14] ПГ. Е. Собол ев ск ий, Обобщенные решения дифференциальных уравне ний первого порлдка в аильбертовом пространстве, Доклады А. Н. СССР 122 (1958), p. 994-996.

[15] А. Д. В ент це ль, О ераничных условиях для многомерных диффузионных прочессов, Теория вер. и её прим. 4 (1959), p. 172-185.

[16] T. Ważuwski, Une généralisation des théorèmes sur les accroissements finis au ras des espaces de Banach et application à la généralisation du théorème do l'Hôpital, Ann. Soc. Polon. Math. 24 (1951), p. 132-147.

[17] K. Yosida, An operator-theoretical treatment of temporaly homogeneous Markoff process, Journ. Math. Soc. Japan 1 (1949), p. 244-253

[18] - On Brownian motion in a homogeneous Riemannian space, Pacific Journ. of Math. 2 (1952), p. $263-270$

[19] I. S. Gál, On the fundamental theorems of the calculus, Trans. Amer. Math. Soc. 86.2 (1957), p. $309-320$

INSTYTUT MATEMATYCZNY POLSKIEJ AKADEMII NAUK

MATHEMATICAL INSTITUTE OF THE POLISH ACADEMY OF SCIENCES

Reģu par la Rédaction le 18. 10. 1959 